\title{
The Correlation between Thyroid Hormone Levels and the Kidney Disease Progression Risk in Patients with Type 2 Diabetes
}

\author{
Zhi Yang ${ }^{1-3}$ \\ Peng Duan ${ }^{2}$ \\ Weihong $\mathrm{Li}^{2}$ \\ Ronghui $\mathrm{Nie}^{2,3}$ \\ Xiaoyang Lou ${ }^{2,4}$ \\ Lina Wang ${ }^{2}$ \\ Kexia $\mathrm{Wu}^{2}$ \\ Jiang $\mathrm{Liu}^{2}$ \\ Ping $\mathrm{Tu}^{2}$ \\ Xiaoyang Lai'
}

'Department of Endocrinology and Metabolism, The Second Affiliated Hospital of Nanchang University, Nanchang, 330008, Jiangxi, People's Republic of China; ${ }^{2}$ Department of Endocrinology and Metabolism, The Third Hospital of Nanchang, Nanchang, 330000, Jiangxi, People's Republic of China; ${ }^{3}$ Jiangxi Medical College of Nanchang University, Nanchang, 330036, Jiangxi, People's Republic of China; ${ }^{4}$ Jiangxi University of Traditional Chinese Medicine, Nanchang, 330004, Jiangxi, People's Republic of China
Objective: We investigated the relationship between thyroid hormones and the risk of diabetic kidney disease (DKD) progression.

Methods: A total of 452 patients with type 2 diabetes were included, and a cross-sectional analysis was performed. Urine albumin/creatinine ratio (ACR) and estimated glomerular filtration rate (eGFR) were used to diagnose persistent albuminuria and stage chronic kidney disease, respectively. The Kidney Disease: Improving Global Outcomes (KDIGO) clinical practice guideline was used to describe the risk of DKD progression (low, moderate, and high or very high risks).

Results: The DKD group had higher levels of thyroid-stimulating hormone (TSH) and lower levels of free triiodothyronine $\left(\mathrm{FT}_{3}\right)$ and free thyroxine $\left(\mathrm{FT}_{4}\right)$ than the non-DKD group. The prevalence of thyroid dysfunction in the DKD group was significantly higher than in the nonDKD group, especially the prevalence of subclinical hypothyroidism. $\mathrm{FT}_{3}$ levels decreased gradually with the deterioration of DKD. TSH levels increased with an increasing KDIGO category. $\mathrm{FT}_{3}$ and $\mathrm{FT}_{4}$ levels were negatively correlated with serum creatinine levels and ACR, and positively correlated with eGFR. Contrastingly, TSH was positively correlated with ACR, and negatively correlated with eGFR. After adjustment, an increase in $\mathrm{FT}_{3}$ levels significantly reduced the risk of DKD [odds ratio, OR ( $95 \%$ confidence interval, $\mathrm{CI})=0.58$ $(0.42-0.79)]$ and DKD progression [ORs $(95 \% \mathrm{CIs})=0.65(0.45-0.93)$ for the moderate risk group and $0.50(0.33-0.74)$ for the high or very high-risk group, using the low-risk group as a reference]. $\mathrm{FT}_{3}$ levels below $4.30 \mathrm{pmol} / \mathrm{L}$ in men and $3.99 \mathrm{pmol} / \mathrm{L}$ in women were the cutoff points for an increased risk of DKD progression.

Conclusion: Low $\mathrm{FT}_{3}$ level is an independent risk factor for DKD and DKD progression. $\mathrm{FT}_{3} \leq 4.30 \mathrm{pmol} / \mathrm{L}$ in men and $\leq 3.99 \mathrm{pmol} / \mathrm{L}$ in women will greatly increase the risk of kidney disease progression in patients with type 2 diabetes.

Keywords: thyroid hormone, prognosis of chronic kidney disease, type 2 diabetes mellitus

\section{Introduction}

Diabetes leads to the damage of several organs and tissues, resulting in chronic complications; kidney disease is one of the common microvascular complications. Approximately $40 \%$ of the patients with diabetes develop chronic kidney disease (CKD). ${ }^{1}$ This increases the risk of end-stage kidney disease and cardiovascular disease, leading to a high mortality. ${ }^{2}$ In patients with type 2 diabetes mellitus (T2DM), the risk of cardiovascular diseases increases by 2-3 times when patients have higher levels of albuminuria, compared with those without significant albuminuria. ${ }^{3}$ Therefore, early
Correspondence: Xiaoyang Lai

$\mathrm{Tel}+86$ I36079/6298

Email1640852510@qq.com 
detection and management of diabetic nephropathy is very important in delaying its progression. Furthermore, it is important to protect the kidneys, in order to significantly reduce the risk of comorbidities.

Thyroid hormones are involved in cell metabolism, blood sugar regulation, and insulin resistance. The prevalence of an abnormal thyroid function is higher in patients with diabetes (approximately $2.2-17 \%$ increase) than in healthy individuals. ${ }^{4}$ Hypothyroidism is the commonest abnormal thyroid function in these patients. ${ }^{5}$ In addition, thyroid hormones interact with the kidneys. ${ }^{6}$ Thyroid hormones contribute to the growth and development of the kidneys, maintain water and electrolyte balance, and participate in the renal transport system. Thyroid dysfunction affects the function of the glomerulus and renal tubules, and indirectly affects the renin-angiotensin system and renal blood flow. Changes in hemodynamics and the cardiovascular system have an effect on renal function. ${ }^{7,8}$ Besides, the kidneys are the target organs for thyroid hormones. They are involved in the metabolism and elimination of thyroid hormones. Chronic kidney disease affects the synthesis of thyroid hormones by regulating the hypothalamus-pituitary-thyroid axis. ${ }^{9,10}$ Previous studies have shown that thyroid hormones can interfere with the accumulation of collagen in the cortical interstitium and glomeruli; ${ }^{11}$ thyroid-stimulating hormone (TSH) influences kidney disease progression; ${ }^{12}$ and low triiodothyronine $\left(T_{3}\right)$ levels may play a role in worsening renal function. ${ }^{13}$ Therefore, the purpose of this study was to explore the correlation between thyroid hormones and the progression risk of type 2 diabetic kidney disease (DKD).

\section{Methods}

The present cross-sectional study analyzed the hospitalization data of 452 subjects with type 2 diabetes Table S1. The risk of DKD progression was evaluated by a combination of urine albumin/creatinine ratio (ACR) and estimated glomerular filtration rate (eGFR).

\section{Study Population}

All subjects of this study were patients with type 2 diabetes aged 18 years or above. They were routinely hospitalized in the Department of Endocrinology and Metabolism of the Third Hospital of Nanchang between February 2018 and November 2020. We tried our best to obtain sufficient informed consent from the subjects and avoid selection bias as much as possible. All subjects participated in the study voluntarily and signed written the informed consent form at admission. The exclusion criteria were as follows: 1) other types of diabetes; 2) taking drugs that affect thyroid hormone levels; 3) urinary tract infections or receiving drugs that affect urine protein; 4) an active urinary sediment (containing red or white blood cells or cellular casts), rapidly increasing albuminuria or nephrotic syndrome, rapidly decreasing eGFR; ${ }^{14}$ 5) multiple hospitalizations. A total of 486 patients were enrolled, and 34 patients with incomplete data were excluded. Finally, 452 patients were included in the study.

\section{Laboratory Assays}

An ultrasonic instrument (Omron HNH-318, Japan) was used to measure the height and weight of each patient to the nearest $0.1 \mathrm{~cm}$ and $0.1 \mathrm{~kg}$, respectively. These values were used to calculate the body mass index (BMI) by the formula, BMI=weight $(\mathrm{kg}) /$ height $^{2}\left(\mathrm{~m}^{2}\right)$.

Venous blood was collected from patients in the morning in an empty stomach (fasting for at least $8 \mathrm{~h}$ ). Fasting blood glucose (FBG), serum creatinine ( $\mathrm{SCr}$ ), blood urea nitrogen (BUN), uric acid (UA), serum total cholesterol (TC), triglycerides (TG), high-density lipoprotein cholesterol (HDL-C), and low-density lipoprotein cholesterol (LDL-C) levels were measured on the same autoanalyzer (Roche, Basel, Switzerland). Glycated hemoglobin (HbA1c) was detected using high-performance liquid chromatography (Bio Rad D-10, Berkeley, USA). Chemiluminescence immunoassay was used to measure serum $\mathrm{TSH}, \mathrm{FT}_{3}$, and $\mathrm{FT}_{4}$ levels (Siemens ADVIA Centaur XP, Germany). The reference ranges of TSH, $\mathrm{FT}_{3}$, and $\mathrm{FT}_{4}$ levels were $0.38-4.34 \mathrm{mIU} / \mathrm{L}, 2.77-6.31 \mathrm{pmol} / \mathrm{L}$ and $10.45-24.38 \mathrm{pmol} / \mathrm{L}$, respectively. Urine albumin levels were measured using the immunological turbidimetry assay, and urine creatinine levels were measured using the picric acid method (Siemens ADVRI 2400, Germany). The urine albu$\mathrm{min} /$ creatinine ratio (ACR) was calculated. The eGFR was calculated using the Chronic Kidney Disease Epidemiology Collaboration (CKD-EPI) equation.

DKD was defined as eGFR $<60 \mathrm{~mL} / \mathrm{min} / 1.73 \mathrm{~m}^{2}$ and/ or ACR $\geq 30 \mathrm{mg} / \mathrm{g} .{ }^{14}$ Patients with or without DKD were grouped. Three different renal function classifications were used for the grouping analysis: 1) ACR $<30,30-300$ and $>300 \mathrm{mg} / \mathrm{g}$ were defined as normal albuminuria, microalbuminuria and massive albuminuria, respectively; 2) eGFR $\geq 90,60-89,45-59,30-44,15-19$ and $<15 \mathrm{~mL} /$ $\mathrm{min} / 1.73 \mathrm{~m}^{2}$ were defined as stages $1,2,3 \mathrm{a}, 3 \mathrm{~b}, 4$ and 5 $\mathrm{CKD}$, respectively; and 3) the Kidney Disease: Improving Global Outcomes (KDIGO) classification, ${ }^{15}$ which 
combined eGFR with ACR, was used to assess the risk of DKD progression (low, moderate, high or very high risks) (Figure 1).

\section{Statistical Analyses}

All analyses were performed using SPSS 25.0. Count data are expressed as rates $(\%)$, and the $\chi^{2}$ test was used for comparison between groups. Normally distributed measurement data are expressed as means \pm standard deviations; the differences between the groups were calculated using one-way analysis of variance. Non-normally distributed data are expressed as medians (interquartile ranges: 25th and 75th percentile); the Mann-Whitney and Kruskal-Wallis tests were used to compare between the groups. The correlation between thyroid hormones and various variables were assessed using Spearman correlation analysis method. A multivariable logistic regression model was used to analyze the association between thyroid function and diabetic nephropathy. The confounding factors included sex, age, FBG, HbA1c, TC, TG, HDL-C and LDL-C. To identify the cut-off points for thyroid hormones in assessing the risk of DKD progression, further subgroup analyses by sex were performed. P values $<0.05$ were considered statistically significant.

\section{Results}

The Clinical Characteristics of Study

Subjects

Among the 452 subjects, 188 (41.6\%) had diabetic nephropathy, 264 (58.4\%) did not have diabetic nephropathy, and there was no significant difference between female and male $(\mathrm{P}>0.05)$. Compared with the patients without DKD, patients with DKD were a little younger, had lower eGFR, $\mathrm{FT}_{3}$ and $\mathrm{FT}_{4}$ levels; and higher BUN, $\mathrm{SCr}$, UA, LDL-C, ACR, and TSH levels $(\mathrm{P}<0.05)$ (Table 1).

\section{The Prevalence of Thyroid Dysfunction in Subjects with or without DKD}

In subjects with DKD, the overall prevalence of thyroid dysfunction (any of hyperthyroidism or subclinical hyperthyroidism or hypothyroidism or subclinical hypothyroidism) was $22.9 \%$, compared with $17.5 \%$ in patients without DKD $(\mathrm{P}=0.009)$. The proportion of subclinical hypothyroidism in DKD group was observed as high as $19.7 \%$, which was significantly different from that in the non-DKD group (9.5\%). (Figure 2)

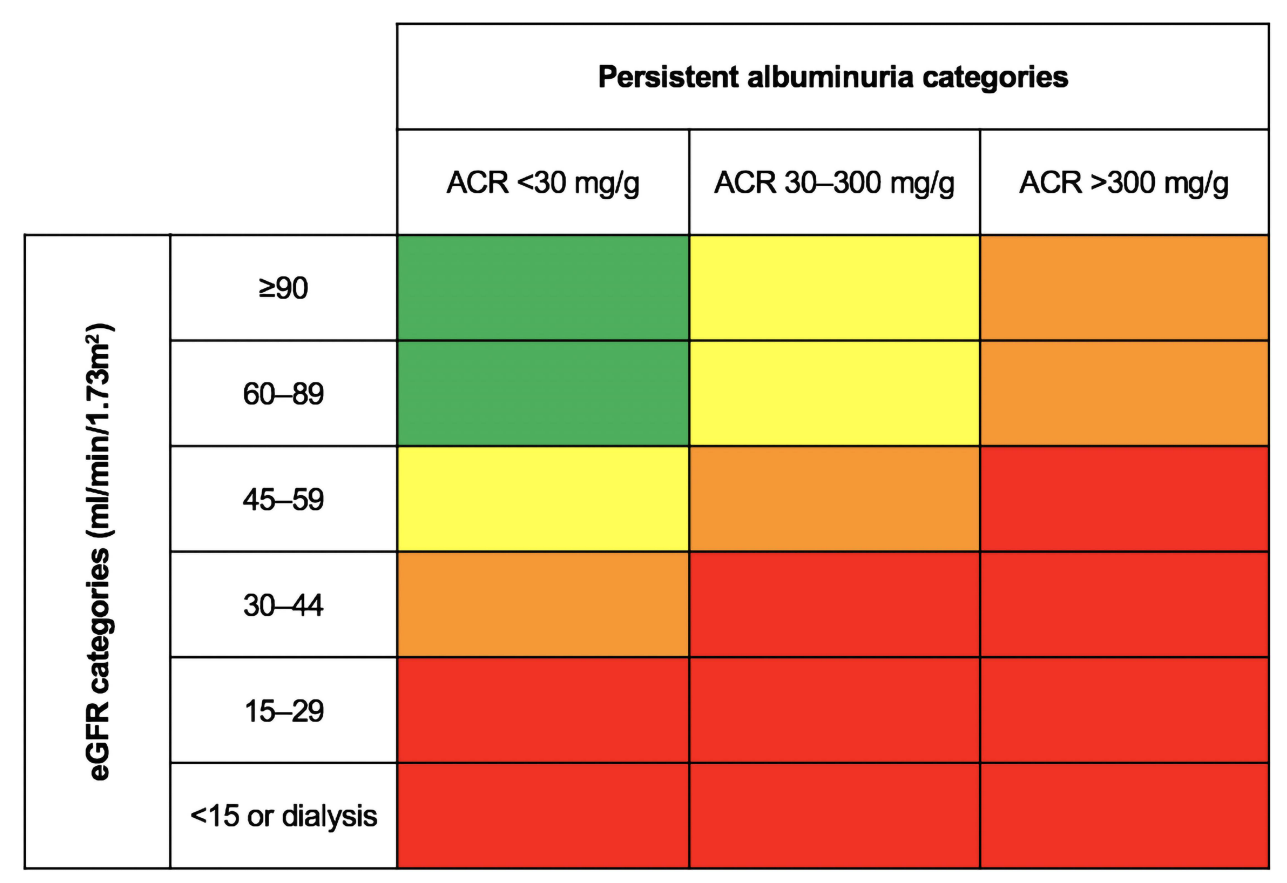

Figure I The KDIGO risk categories.

Notes: Green, low risk; yellow, moderate risk; orange, high risk; red, very high risk.

Abbreviations: ACR, albumin/creatinine ratio; eGFR, estimated glomerular filtration rate; KDIGO, Kidney Disease: Improving Global Outcomes. 
Table I The Clinical Characteristics of Study Subjects with or without DKD

\begin{tabular}{|c|c|c|c|}
\hline Variables & $\begin{array}{l}\text { Patients without DKD } \\
\qquad(n=264)\end{array}$ & $\begin{array}{l}\text { Patients with DKD } \\
(\mathrm{n}=188)\end{array}$ & P value \\
\hline Sex (n, female/male) & $121 / 143$ & $92 / 96$ & 0.515 \\
\hline Age (years) & $65.09 \pm 11.62$ & $63.15 \pm 11.91$ & $<0.001$ \\
\hline BMI $\left(\mathrm{kg} / \mathrm{m}^{2}\right)$ & $24.58(22.48,26.59)$ & $24.90(22.63,27.62)$ & 0.063 \\
\hline $\mathrm{FBG}(\mathrm{mmol} / \mathrm{L})$ & $8.20(6.60,11.08)$ & $8.52(6.40,11.18)$ & 0.763 \\
\hline HbAlc (\%) & $8.5(6.8,10.7)$ & $8.8(7.1,10.7)$ & 0.328 \\
\hline BUN (mmol/L) & $5.10(4.20,5.97)$ & $6.00(4.67,8.18)$ & $<0.001$ \\
\hline $\mathrm{SCr}(\mu \mathrm{mol} / \mathrm{L})$ & $64(54,76)$ & $81(60,112)$ & $<0.001$ \\
\hline $\mathrm{UA}(\mu \mathrm{mol} / \mathrm{L})$ & $275(228,334)$ & $318(256,391)$ & $<0.001$ \\
\hline TG (mmol/L) & $1.34(1.02,2.19)$ & $1.53(1.04,2.30)$ & 0.280 \\
\hline $\mathrm{TC}(\mathrm{mmol} / \mathrm{L})$ & $4.45(3.84,5.31)$ & $4.40(3.66,5.15)$ & 0.280 \\
\hline HDL-C (mmol/L) & $1.22(1.06,1.43)$ & $1.20(1.00,1.41)$ & 0.166 \\
\hline LDL-C (mmol/L) & $2.63(2.13,3.33)$ & $2.54(1.96,3.14)$ & 0.048 \\
\hline eGFR $\left(\mathrm{mL} / \mathrm{min} / \mathrm{I} .73 \mathrm{~m}^{2}\right)$ & $107.28 \pm 39.47$ & $84.21 \pm 48.98$ & $<0.001$ \\
\hline ACR (mg/g) & $12.48(8.53,18.30)$ & $128.40(49.17,444.75)$ & $<0.001$ \\
\hline $\mathrm{FT}_{3}(\mathrm{pmol} / \mathrm{L})$ & $4.52(4.13,4.84)$ & $4.15(3.72,4.66)$ & $<0.001$ \\
\hline $\mathrm{FT}_{4}(\mathrm{pmol} / \mathrm{L})$ & $16.82(15.42,18.34)$ & $16.52(14.73,18.04)$ & 0.038 \\
\hline TSH (mlU/L) & $2.03(1.34,2.97)$ & $2.46(1.42,3.86)$ & 0.013 \\
\hline
\end{tabular}

Notes: Data are presented as mean \pm standard deviations or medians ( 25 th, 75 th percentile) or numbers. DKD was defined as eGFR $<60 \mathrm{~mL} / \mathrm{min} / \mathrm{l} .73 \mathrm{~m}{ }^{2}$ and/or ACR $\geq 30 \mathrm{mg} / \mathrm{g}$. Abbreviations: DKD, diabetic kidney disease; BMI, body mass index; FBG, fasting blood glucose; HbAlc, glycated hemoglobin; BUN, blood urea nitrogen; SCr, serum creatinine; UA, uric acid; TG, triglycerides; TC, total cholesterol; HDL-C, high-density lipoprotein cholesterol; LDL-C, low-density lipoprotein cholesterol; FT 3 , free triiodothyronine; $\mathrm{FT}_{4}$, free thyroxine; $\mathrm{TSH}$, thyroid-stimulating hormone.

\section{Comparison of Thyroid Hormone Levels in Different DKD Categories}

Due to the small numbers of people in stages 4 and 5 $\mathrm{CKD}$, we combined the two groups into eGFR $<30 \mathrm{~mL} /$ $\min / 1.73 \mathrm{~m}^{2}$. The results showed that $\mathrm{FT}_{3}$ levels varied with degrees of albuminuria, kidney damage, and KDIGO risk $(\mathrm{P}<0.05)$, and decreased with increasing severity. $\mathrm{FT}_{4}$ levels decreased with worsening $\mathrm{CKD}$ stages, and TSH levels increased with a higher risk of KDIGO categories $(\mathrm{P}<0.05)$ (Table 2).

\section{Correlation Analysis Between Thyroid Hormones and Indexes of Renal Function}

There was a negative correlation between $\mathrm{FT}_{3}$ and $\mathrm{FT}_{4}$ levels with both $\mathrm{SCr}$ and $\mathrm{ACR}(r=-0.168, \mathrm{P}<0.001$ and $r=-0.107, \mathrm{P}=0.023 ; r=-0.267, \mathrm{P}<0.001$ and $r=-0.109$, $\mathrm{P}=0.021$, respectively). Meanwhile, $\mathrm{FT}_{3}$ and $\mathrm{FT}_{4}$ levels were positively correlated with eGFR $(r=0.325, \mathrm{P}<0.001$ and $r=0.165, \mathrm{P}<0.001$, respectively). In contrast, there was a negative correlation between TSH levels and eGFR ( $r=-0.128, \mathrm{P}=0.006$ ), and a positive correlation between TSH levels and ACR $(r=0.104, \quad \mathrm{P}=0.027)$ (Table 3).

\section{Regression Analysis of Thyroid Hormones and the Risk of Diabetic Nephropathy Progression}

A logistic regression analysis was performed, using with or without diabetic nephropathy as the dependent variable and using thyroid hormone levels as the independent variable. After adjusting for sex, age, FBG, HbAlc, TC, TG, HDL-C and LDL-C, an increase in $\mathrm{FT}_{3}$ levels significantly reduced the risk of having diabetic nephropathy [odds ratio, OR $(95 \%$ confidence interval, CI $)=0.58 \quad(0.42-0.79), \quad \mathrm{P}=0.001]$. However, $\mathrm{FT}_{4}$ and TSH levels did not influence the risk of disease [OR $(95 \% \mathrm{CI})$ of $\mathrm{FT}_{4}=0.97(0.92-1.02), \mathrm{P}=0.281$; OR $(95 \%$ CI) of $\mathrm{TSH}=1.02 \quad(0.99-1.05), \mathrm{P}=0.147]$ (Table 4).

The risk of diabetic nephropathy progression (KDIGO risk categories) was further used as the dependent variable. When using the low KDIGO risk group as the reference group, an increase in $\mathrm{FT}_{3}$ level significantly reduced the risk of DKD progression [moderate risk, OR $(95 \% \mathrm{CI})=0.65(0.45-0.93), \mathrm{P}=0.020$; high or very high risk, $\mathrm{OR} \quad(95 \% \quad \mathrm{CI})=0.50 \quad(0.33-0.74)$, $\mathrm{P}=0.001]$ (Table 5). 
A

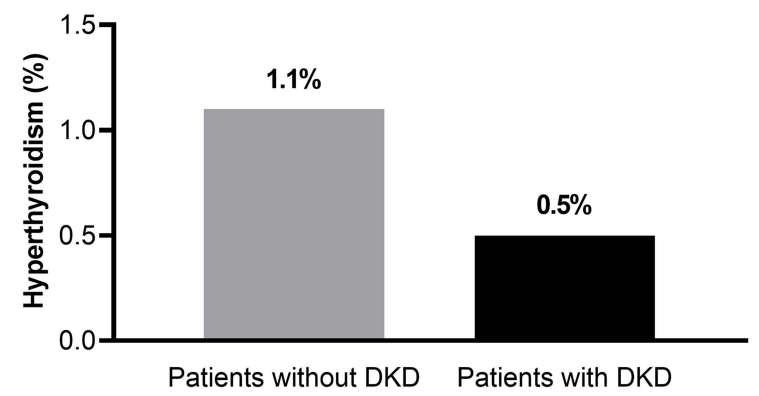

C

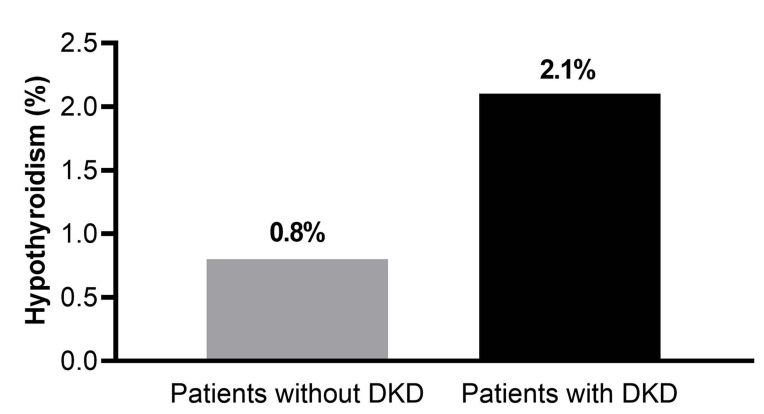

B

$P=0.248$

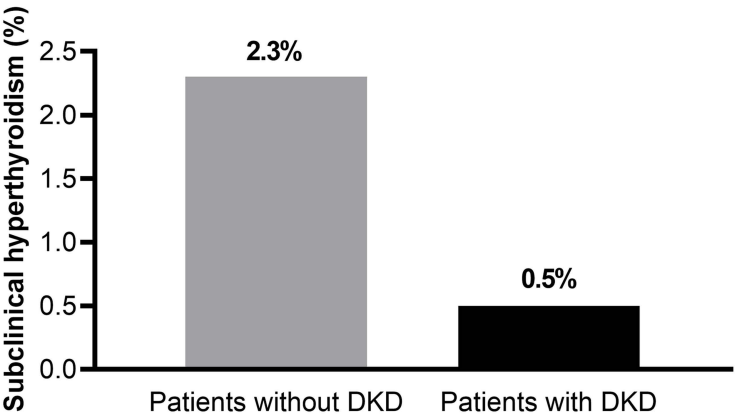

D

$P=0.002$

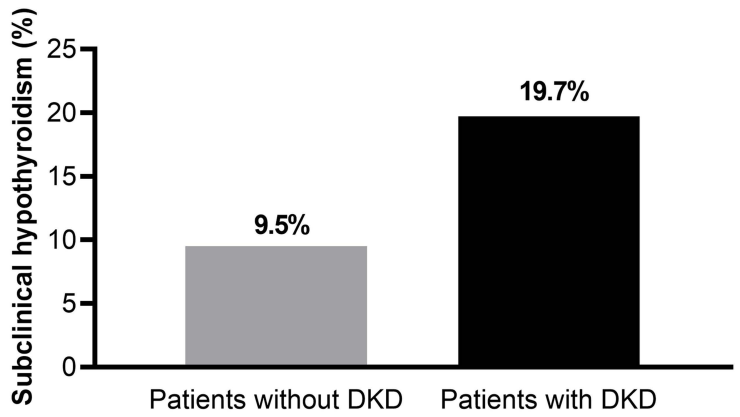

Figure 2 The prevalence of thyroid dysfunction in subjects with or without DKD.

Notes: A, B, C and D represent the prevalence of hyperthyroidism, subclinical hyperthyroidism, hypothyroidism, and subclinical hypothyroidism respectively.

Subgroup Analysis of $\mathrm{FT}_{3}$ and the Risk of Diabetic Nephropathy Progression

Further, the KDIGO risk categories were the dependent variables in the subgroup analysis (reference: the low-risk group), and the tri-sectional quantiles of $\mathrm{FT}_{3}$ were the independent variables (reference: the highest tertile). The results showed that the lowest tertile of $\mathrm{FT}_{3}$ not only increased the high or very high risk of DKD progression in men [OR $(95 \% \mathrm{CI})=3.66(1.54-8.71), \mathrm{P}=0.003$ ] but also increased all risks of DKD progression in women

Table 2 Thyroid Hormone Levels in Different ACR, eGFR and KDIGO Risk Categories

\begin{tabular}{|c|c|c|c|c|}
\hline \multicolumn{2}{|l|}{ Categories (n, \%) } & \multirow{2}{*}{$\frac{\mathbf{F T}_{\mathbf{3}}(\mathrm{pmol} / \mathrm{L})}{4.51(4.12,4.83)}$} & \multirow{2}{*}{$\frac{\mathbf{F T}_{\mathbf{4}}(\mathbf{p m o l} / \mathbf{L})}{16.76(15.43,18.32)}$} & \multirow{2}{*}{$\frac{\text { TSH }(\mathrm{mIU} / \mathrm{L})}{2.06(1.35,3.0 \mathrm{I})}$} \\
\hline$A C R$ & Normal albuminuria $(267,59.1 \%)$ & & & \\
\hline & Microalbuminuria $(120,26.5 \%)$ & $4.18(3.75,4.66)$ & $16.61(14.80,18.32)$ & $2.45(1.42,3.76)$ \\
\hline & Massive albuminuria $(65,14.4 \%)$ & $4.10(3.55,4.68)$ & $16.52(14.19,17.74)$ & $2.44(1.39,3.96)$ \\
\hline & $P$ value & $<0.001$ & 0.062 & 0.094 \\
\hline \multirow[t]{6}{*}{ eGFR $\left(\mathrm{mL} / \mathrm{min} / 1.73 \mathrm{~m}^{2}\right)$} & $\geq 90(332,73.4 \%)$ & $4.49(4.11,4.83)$ & $16.72(15.27,18.34)$ & $2.16(1.42,3.29)$ \\
\hline & $60-89(78,17.3 \%)$ & $4.23(3.75,4.62)$ & $16.72(15.52,18.23)$ & $1.86(1.28,3.35)$ \\
\hline & $45-59(22,4.9 \%)$ & $3.82(3.70,4.03)$ & $16.18(14.01,18.60)$ & $3.18(1.73,4.83)$ \\
\hline & $30-44(10,2.2 \%)$ & $3.55(3.15,5.26)$ & $16.29(11.44,16.73)$ & $1.32(0.98,3.11)$ \\
\hline & $<30(10,2.2 \%)$ & $3.34(2.75,4.12)$ & $14.42(13.07,16.32)$ & $2.14(1.18,4.27)$ \\
\hline & $P$ value & $<0.001$ & 0.011 & 0.088 \\
\hline \multirow[t]{4}{*}{ KDIGO categories } & Low risk $(264,58.4 \%)$ & $4.52(4.13,4.84)$ & $16.82(15.54,18.34)$ & $2.03(1.34,2.97)$ \\
\hline & Moderate risk $(109,24.1 \%)$ & $4.22(3.78,4.7 I)$ & $16.52(15.14,18.12)$ & $2.44(1.46,3.50)$ \\
\hline & High or very high risk $(79,17.5 \%)$ & $3.97(3.56,4.55)$ & $16.52(13.92,17.93)$ & $2.58(1.38,4.41)$ \\
\hline & $P$ value & $<0.001$ & 0.05 & 0.045 \\
\hline
\end{tabular}

Note: Data are presented as numbers (proportions) or medians (25th, 75 th percentile). 
Table 3 Correlation Between Thyroid Hormones and Parameters of Renal Function

\begin{tabular}{|l|c|c|c|}
\hline \multicolumn{2}{|l|}{ Parameters } & r value & P value \\
\hline $\mathrm{FT}_{3}$ with & SCr & -0.168 & $<0.001$ \\
& eGFR & 0.325 & $<0.001$ \\
& ACR & -0.267 & $<0.001$ \\
\hline $\mathrm{FT}_{4}$ with & SCr & -0.107 & 0.023 \\
& eGFR & 0.165 & $<0.001$ \\
& ACR & -0.109 & 0.021 \\
\hline \multirow{2}{*}{ TSH with } & SCr & 0.043 & 0.359 \\
& eGFR & -0.128 & 0.006 \\
& ACR & 0.104 & 0.027 \\
\hline
\end{tabular}

Table 4 Logistic Regression Analysis of Thyroid Hormones and the Risk of Having Diabetic Nephropathy

\begin{tabular}{|l|c|c|c|c|c|}
\hline Variable & B & SE & Wald $\mathbf{X}^{\mathbf{2}}$ & OR (95\% Cl) & P value \\
\hline $\mathrm{FT}_{3}$ & -0.55 & 0.16 & 11.58 & $0.58(0.42-0.79)$ & 0.001 \\
$\mathrm{FT}_{4}$ & -0.03 & 0.03 & 1.16 & $0.97(0.92-1.02)$ & $0.28 \mathrm{I}$ \\
$\mathrm{TSH}$ & 0.02 & 0.02 & 2.11 & $1.02(0.99-1.05)$ & 0.147 \\
\hline
\end{tabular}

Note: All models were adjusted for sex, age, FBG, HbAIc, TC, TG, HDL-C and LDL-C.

Table 5 Logistic Regression Analysis of Thyroid Hormones and the Risk of Diabetic Nephropathy Progression

\begin{tabular}{|l|c|c|c|c|}
\hline \multirow{2}{*}{ Variable } & \multicolumn{2}{|c|}{ Moderate KDIGO Risk } & \multicolumn{2}{c|}{ High or Very High KDIGO Risk } \\
\cline { 2 - 5 } & OR (95\% Cl) & P value & OR (95\% CI) & P value \\
\hline $\mathrm{FT}_{3}$ & $0.65(0.45-0.93)$ & 0.020 & $0.50(0.33-0.74)$ & 0.001 \\
$\mathrm{FT}_{4}$ & $0.98(0.92-1.04)$ & 0.476 & $0.96(0.89-1.04)$ & 0.332 \\
$\mathrm{TSH}$ & $1.02(0.99-1.06)$ & 0.119 & $1.02(0.98-1.05)$ & 0.333 \\
\hline
\end{tabular}

Notes: The low KDIGO risk group was classified as the reference group. All models were adjusted for sex, age, FBG, HbAlc, TC, TG, HDL-C and LDL-C.

[moderate risk, OR $(95 \% \mathrm{CI})=2.37(1.02-5.51), \mathrm{P}=0.044$; high or very high risk, OR $(95 \% \mathrm{CI})=7.23(1.72-30.34)$, $\mathrm{P}=0.007]$. Therefore, we obtained two cut-off points for $\mathrm{FT}_{3}$ to assess the increased risk of DKD progression, which were less than $4.30 \mathrm{pmol} / \mathrm{L}$ for men and less than $3.99 \mathrm{pmol} / \mathrm{L}$ for women, respectively (Table 6).

\section{Discussion}

Our results showed that $\mathrm{FT}_{3}$ and $\mathrm{FT}_{4}$ were negatively correlated with $\mathrm{SCr}$ and $\mathrm{ACR}$ and positively correlated with eGFR. In contrast, TSH was negatively correlated with eGFR and positively correlated with ACR. These findings were consistent with previous studies ${ }^{16-18}$ and suggested that thyroid hormones were related to renal function in patients with diabetes. Firstly, thyroid hormones were involved in glucose metabolism, and the prevalence of thyroid dysfunction in patients with diabetes was higher than that in healthy individuals. ${ }^{19}$ Secondly, our further findings showed that $\mathrm{FT}_{3}$ levels decreased gradually with increased severity of kidney damage and albuminuria. Even with normal thyroid function, $\mathrm{FT}_{3}$ was positively correlated with eGFR and negatively correlated with ACR in previous study. ${ }^{18}$ Thirdly, TSH levels increased and $\mathrm{FT}_{3}$ levels decreased in higher risk groups of DKD progression, using the KDIGO classification. Previous studies suggested that patients with diabetes who had higher TSH levels and lower $\mathrm{FT}_{3}$ levels were more likely to develop DKD. ${ }^{12,20}$ In addition, a higher prevalence of subclinical hypothyroidism was found in the DKD group. Subclinical hypothyroidism was regarded as an independent risk factor for $\mathrm{DKD},{ }^{21,22}$ and thyroid replacement therapy can play a role in renal protection. $^{23,24}$ 
Table 6 Subgroup Analysis of $\mathrm{FT}_{3}$ and Risk of DKD Progression Stratified by Male and Female

\begin{tabular}{|l|c|c|c|c|c|}
\hline \multirow{2}{*}{ Subgroup } & Ranges of $\mathbf{F T}_{\mathbf{3}}$ (pmol/L) & \multicolumn{2}{|c|}{ Moderate KDIGO Risk } & \multicolumn{2}{c|}{ High or Very High KDIGO Risk } \\
\cline { 3 - 6 } & & OR (95\% CI) & P value & OR (95\% CI) & P value \\
\hline \multirow{2}{*}{ Male } & $>4.78$ & 1.0 & - & 1.0 & - \\
& $4.31-4.78$ & $1.10(0.45-2.64)$ & 0.838 & $0.62(0.24-1.61)$ & 0.325 \\
& $\leq 4.30$ & $1.93(0.74-5.04)$ & 0.181 & $3.66(1.54-8.71)$ & 0.003 \\
\hline \multirow{2}{*}{ Female } & $>4.44$ & 1.0 & - & 1.0 & - \\
& $4.00-4.44$ & $1.36(0.60-3.04)$ & $0.46 I$ & $1.49(0.32-7.01)$ & 0.614 \\
& $\leq 3.99$ & $2.37(I .02-5.5 I)$ & 0.044 & $7.23(1.72-30.34)$ & 0.007 \\
\hline
\end{tabular}

Notes: The levels of $\mathrm{FT}_{3}$ were divided into tri-sectional quantiles. The low KDIGO risk group and the highest tertile of $\mathrm{FT}_{3}$ were classified as the reference groups of dependent variable and independent variable, respectively. All models were adjusted for age, FBG, HbAIc, TC, TG, HDL-C, and LDL-C.

An insufficiency of the thyroid gland may be related to intrarenal vasoconstriction, a decrease in cardiac output, and a decrease in effective renal blood flow, which cause the final results of renal damage and an appearance of microalbuminuria. ${ }^{25,26}$ In addition, Reinhardt et $\mathrm{al}^{27}$ showed that the severity of albuminuria in patients with CKD had the greatest impact on the concentration of reverse triiodothyronine $\left(\mathrm{rT}_{3}\right)$, and the serum $\mathrm{rT}_{3}$ concentration was significantly negatively correlated with the degree of albuminuria. Furthermore, because most thyroid hormones bind to proteins, protein loss caused by albuminuria will lead the depletion of thyroid hormones, which in turn stimulates the hypothalamus-pituitary-thyroid axis through negative feedback and inhibits the conversion of $\mathrm{T}_{4}$ to $\mathrm{T}_{3}$ in peripheral tissues, resulting in an increase in the TSH level and a decrease in the $\mathrm{T}_{3}$ level. $^{28}$

In order to confirm the strong association between thyroid hormones and DKD, a logistic regression analysis was performed. Our study suggested that the increase in $\mathrm{FT}_{3}$ level significantly reduced the risks of DKD and DKD progression by about $35-50 \%$. Consistently with other studies, ${ }^{29-32}$ low $\mathrm{T}_{3}$ level was disclosed as a common feature in patients with kidney disease, and $\mathrm{FT}_{3}$ could be an effective marker for predicting DKD and evaluating prognosis.

The role of $\mathrm{FT}_{3}$ in diabetic nephropathy progression is unclear. However, there are some hypotheses. Firstly, endothelial dysfunction and podocyte disease play a key role in the pathogenesis and development of DKD. ${ }^{33}$ Microalbuminuria is a sign of systemic endothelial dysfunction and vascular damage, ${ }^{34}$ and thyroid hormones can affect vasodilation, regulate endothelial function and homeostatic signal transduction processes. ${ }^{35}$ Experimental models have confirmed that $T_{3}$ can directly or indirectly relax vascular smooth muscle cells, thereby affecting endothelial function. ${ }^{36}$ In patients with stage 3-4 CKD without diabetes, it was also proven that low serum $\mathrm{FT}_{3}$ level was related to endothelial dysfunction assessed by the blood flow-mediated expansion method. ${ }^{37}$ On the contrary, sufficient $T_{3}$ can promote podocyte redifferentiation, reduce hypertrophy, and improve the kidney structure. ${ }^{38}$ Secondly, hyperglycemia can lead to an increase of renal cell cytoplasmic glucose concentration, which leads to the activation of various signaling pathways and oxidative stress. ${ }^{39}$ However, $\mathrm{T}_{3}$ can reduce renal cortex collagen accumulation and glomerular matrix expansion in $\mathrm{db} / \mathrm{db}$ mice, improve kidney damage in diabetic mice, increase renal PI3K activity, and reduce hyperglycemia. Besides, $T_{3}$ can reduce renal transforming growth factor- $\beta 1$ expression, promote insulin synthesis and release, enhance insulin signal transduction, and improve insulin resistance. ${ }^{11}$ Therefore, low $\mathrm{T}_{3}$ levels may indirectly aggravate DKD progression by concomitant hyperglycemia. Thirdly, it was confirmed in an animal study ${ }^{40}$ that overexpressed sirtuin 1 (SIRT1) in podocytes and renal tubular cells can reduce albuminuria and kidney damage. 3,5-diiodothyronine, the natural metabolite of $\mathrm{T}_{3}$ in the deiodination pathway, can prevent a significant decrease in the activity of SIRT1 in the kidneys of diabetic rats, and can inhibit renal tubular epithelium cell nuclear factor- $\mathrm{\kappa B}$ acetylation and c-Jun N-terminal kinase phosphorylation, which ultimately play a role in protecting the kidneys. Fourthly, inflammation seriously interferes with the thyroid function in subjects with CKD. In these patients, an independent negative correlation between inflammatory cytokines (tumor necrosis factor- $\alpha$, interleukin-6, and C-reactive protein) and $\mathrm{FT}_{3}$ was observed. $^{41,42}$

Beyond doubt, similar results were obtained in both men and women in the subgroup analyses. More seriously, women with a low $\mathrm{FT}_{3}$ level had a maximum risk of DKD progression by 7.23 times. The clinical significance of the 
results was to set the low $\mathrm{FT}_{3}$ threshold to predict the prognosis of chronic kidney disease, which was less than $4.30 \mathrm{pmol} / \mathrm{L}$ for men and less than $3.99 \mathrm{pmol} / \mathrm{L}$ for women, respectively.

This study provides new evidence for the use of low $\mathrm{FT}_{3}$ in evaluating the prognosis of diabetic nephropathy. However, this study has some limitations. First, this was a cross-sectional study, and causality could not be established. Further prospective and longitudinal studies should be conducted. Second, the thyroid autoantibodies of the patients were not evaluated. Studies have shown that anti-thyroid peroxidase antibodies may be related to endothelial dysfunction and subsequent microalbuminuria. ${ }^{43}$ Third, the study population consisted of hospitalized patients with type 2 diabetes; hence, the results could not represent the overall population.

\section{Conclusions}

The results of this study indicated that low $\mathrm{FT}_{3}$ level was an independent risk factor for having DKD and DKD progression. Low $\mathrm{FT}_{3}$ levels, which are less than 4.30 $\mathrm{pmol} / \mathrm{L}$ in men and less than $3.99 \mathrm{pmol} / \mathrm{L}$ in women will greatly increase the risk of kidney disease progression in patients with type 2 diabetes.

\section{Data Sharing Statement}

All data included in this study are available upon request by contact with the corresponding author.

\section{Ethics Approval and Consent to Participate}

The study protocol was approved by the ethics committee of the Third Hospital of Nanchang. The written informed consent was obtained from each subject. The study was carried out in conformity to the Declaration of Helsinki (as revised in 2013).

\section{Author Contributions}

ZY, PD, WL, JL, PT contributed to the conception and design of the study. ZY, WL, RN, XL (X. Lou), LW and $\mathrm{KW}$ participated in data collection. ZY and WL were responsible for data analysis and drafting manuscript. PD and XL (X. Lai) critically revised the manuscript. All authors contributed to data analysis, drafting or revising the article, have agreed on the journal to which the article was submitted, gave final approval of the version to be published, and agree to be accountable for all aspects of the work.

\section{Funding}

This work was supported by the National Natural Science Foundation of China [grant number 81760153, Recipient: Peng Duan], the Key Research and Development Programs by Science and Technology Department of Jiangxi Province [grant numbers 20171BBG70058, 20171ACH80002 and 20181BBG70014, Recipient: Zhi Yang, Ping $\mathrm{Tu}$ and Jiang Liu], the Science and Technology Support Project by Science and Technology Department of Nanchang City [grant number [2020]133, Recipient: Peng Duan].

\section{Disclosure}

The authors declare that they have no conflicts of interest for this work.

\section{References}

1. Alicic RZ, Rooney MT, Tuttle KR. Diabetic kidney disease: challenges, progress, and possibilities. Clin J Am Soc Nephrol. 2017;12 (12):2032-2045. doi:10.2215/CJN.11491116

2. Ferdinando CS, Luca DN, Ornella C, et al. Cardiovascular risk factors and disease management in type 2 diabetic patients with diabetic nephropathy. Diabetes Care. 2006;29(3):498-503. doi:10.2337/diacare.29.03.06.dc051776

3. Stehouwer CD, Gall MA, Twisk JW, et al. Increased urinary albumin excretion, endothelial dysfunction, and chronic low-grade inflammation in type 2 diabetes: progressive, interrelated, and independently associated with risk of death. Diabetes. 2002;51(4):1157-1165. doi:10.2337/diabetes.51.4.1157

4. Papazafiropoulou A, Sotiropoulos A, Kokolaki A, et al. Prevalence of thyroid dysfunction among Greek type 2 diabetic patients attending an outpatient clinic. J Clin Med Res. 2010;2(2):75-78. doi:10.4021/ jocmr2010.03.281w

5. Distiller LA, Polakow ES, Joffe BI. Type 2 diabetes mellitus and hypothyroidism: the possible influence of metformin therapy. Diabet Med. 2014;31(2):172-175. doi:10.1111/dme.12342

6. Iglesias P, Bajo MA, Selgas R, et al. Thyroid dysfunction and kidney disease: an update. Rev Endocr Metab Disord. 2017;18(1):131-144. doi:10.1007/s11154-016-9395-7

7. Meuwese CL, Carrero JJ. Chronic kidney disease and hypothalamicpituitary axis dysfunction: the chicken or the egg? Arch Med Res. 2013;44(8):591-600. doi:10.1016/j.arcmed.2013.10.009

8. Iglesias P, Díez JJ. Thyroid dysfunction and kidney disease. Eur $J$ Endocrinol. 2009;160(4):503-515. doi:10.1530/EJE-08-0 837

9. Jusufovic S, Hodzic E. Functional thyroid disorders are more common in patients on chronic hemodialysis compared with the general population. Mater Sociomed. 2011;23(4):206-209. doi:10.5455/ msm.2011.23.206-209

10. Kaptein EM. Thyroid hormone metabolism and thyroid diseases in chronic renal failure. Endocr Rev. 1996;17(1):45-63. doi:10.1210/edrv$17-1-45$

11. Lin Y, Sun ZJ. Thyroid hormone ameliorates diabetic nephropathy in a mouse model of type II diabetes. J Endocrinol. 2011;209(2):185191. doi:10.1530/JOE-10-0340

12. Sun MT, Hsiao FC, Su SC, et al. Thyrotropin as an independent factor of renal function and chronic kidney disease in normoglycemic euthyroid adults. Endocr Res. 2012;37(3):110-116. doi:10.3109/ 07435800.2011.640374 
13. Ulla TS, Natalie D, Morgan EG, et al. Thyroid function, reduced kidney function and incident chronic kidney disease in a communitybased population: the atherosclerosis risk in communities study. Nephrol Dial Transplant. 2017;32(11):1874-1881. doi:10.1093/ndt/ gfw301

14. American Diabetes Association. 11. Microvascular complications and foot care: standards of medical care in diabetes-2021. Diabetes Care. 2021;44(Suppl1):S151-S167. doi:10.2337/dc21-S011.

15. National Kidney Foundation. KDIGO 2012 clinical practice guideline for the evaluation and management of chronic kidney disease. Kidney Int. 2013;3(1):5-14.

16. Chen Y, Zhang W, Wang NJ, et al. Thyroid parameters and kidney disorder in type 2 diabetes: results from the METAL study. $J$ Diabetes Res. 2020;2020:4798947. doi:10.1155/2020/4798947

17. Zhao W, Li X, Liu X, et al. Thyroid function in patients with type 2 diabetes mellitus and diabetic nephropathy: a single center study. $J$ Thyroid Res. 2018;2018:9507028. doi:10.1155/2018/9507028

18. Wu J, Li X, Tao Y, et al. Free triiodothyronine levels are associated with diabetic nephropathy in euthyroid patients with type 2 diabetes. Int J Endocrinol. 2015;2015:204893. doi:10.1155/2015/204893

19. Biondi B, Kahaly GJ, Robertson RP. Thyroid dysfunction and diabetes mellitus: two closely associated disorders. Endocr Rev. 2019;40 (3):789-824. doi:10.1210/er.2018-00163

20. Bjørn OA, Trine B, Lars JV. Association of thyroid function with estimated glomerular filtration rate in a population-based study: the HUNT study. Eur J Endocrinol. 2011;164(1):101-105. doi:10.1530/ EJE-10-0705

21. El-Eshmawy MM, Abd El-Hafez HA, El Shabrawy WO, et al. Subclinical hypothyroidism is independently associated with microalbuminuria in a cohort of prediabetic Egyptian adults. Diabetes Metab J. 2013;37(6):450-457. doi:10.4093/dmj.2013.37.6.450

22. Yasuda T, Kaneto H, Kuroda A, et al. Subclinical hypothyroidism is independently associated with albuminuria in people with type 2 diabetes. Diabetes Res Clin Pract. 2011;94(3):e75-7. doi:10.1016/j. diabres.2011.08.019

23. Shin DH, Lee MJ, Kim SJ, et al. Preservation of renal function by thyroid hormone replacement therapy in chronic kidney disease patients with subclinical hypothyroidism. J Clin Endocrinol Metab. 2012;97(8):2732-2740. doi:10.1210/jc.2012-1663

24. Liu P, Liu R, Chen X, et al. Can levothyroxine treatment reduce urinary albumin excretion rate in patients with early type 2 diabetic nephropathy and subclinical hypothyroidism? A randomized doubleblind and placebo-controlled study. Curr Med Res Opin. 2015;31 (12):2233-2240. doi:10.1185/03007995.2015.1094044

25. Mariani LH, Berns JS. The renal manifestations of thyroid disease. $J$ Am Soc Nephrol. 2012;23(1):22-26. doi:10.1681/ASN.2010070766

26. Quesada A, Sainz J, Wangensteen R, et al. Nitric oxide synthase activity in hyperthyroid and hypothyroid rats. Eur $J$ Endocrinol. 2002;147(1):117-122. doi:10.1530/eje.0.1470117

27. Reinhardt W, Mülling N, Behrendt S, et al. Association between albuminuria and thyroid function in patients with chronic kidney disease. Endocrine. 2021;73(2):367-373. doi:10.1007/s12020-021-02640-1

28. Udiong CE, Udoh AE, Etukudoh ME. Evaluation of thyroid function in diabetes mellitus in Calabar, Nigeria. Indian $J$ Clin Biochem. 2007;22(2):74-78. doi:10.1007/BF02913318
29. Zou J, Tian F, Zhang Y, et al. Association between thyroid hormone levels and diabetic kidney disease in euthyroid patients with type 2 diabetes. Sci Rep. 2018;8(1):4728. doi:10.1038/s41598-018-22904-7

30. Fei X, Xing M, Wo M, et al. Thyroid stimulating hormone and free triiodothyronine are valuable predictors for diabetic nephropathy in patient with type 2 diabetes mellitus. Ann Transl Med. 2018;6 (15):305. doi:10.21037/atm.2018.07.07

31. Yang JW, Han ST, Song SH, et al. Serum $\mathrm{T}_{3}$ level can predict cardiovascular events and all-cause mortality rates in CKD patients with proteinuria. Ren Fail. 2012;34(3):364-372. doi:10.3109/ 0886022X.2011.647342

32. Zhou Y, Ye L, Wang T, et al. Free triiodothyronine concentrations are inversely associated with microalbuminuria. Int $J$ Endocrinol. 2014;2014:959781. doi:10.1155/2014/959781

33. Zoja C, Xinaris C, Macconi D. Diabetic nephropathy: novel molecular mechanisms and therapeutic targets. Front Pharmacol. 2020;11:586892. doi:10.3389/fphar.2020.586892

34. Deckert T, Feldt-Rasmussen B, Borch-Johnsen K, et al. Albuminuria reflects widespread vascular damage. The Steno hypothesis. Diabetologia. 1989;32(4):219-226. doi:10.1007/BF00285287

35. Fukuyama K, Ichiki T, Takeda K, et al. Downregulation of vascular angiotensin II type 1 receptor by thyroid hormone. Hypertension. 2003;41(3):598-603. doi:10.1161/01.HYP.0000056524.35294.80

36. Ojamaa K, Klemperer JD, Klein I. Acute effects of thyroid hormone on vascular smooth muscle. Thyroid. 1996;6(5):505-512. doi:10.1089/thy.1996.6.505

37. Yilmaz MI, Sonmez A, Karaman M, et al. Low triiodothyronine alters flow-mediated vasodilatation in advanced nondiabetic kidney disease. Am J Nephrol. 2011;33(1):25-32. doi:10.1159/000322581

38. Benedetti V, Lavecchia AM, Locatelli M, et al. Alteration of thyroid hormone signaling triggers the diabetes-induced pathological growth, remodeling, and dedifferentiation of podocytes. JCI Insight. 2019;4 (18):e130249. doi:10.1172/jci.insight.130249

39. Al-Kafaji G, Malik AN. Hyperglycemia induces elevated expression of thyroid hormone binding protein in vivo in kidney and heart and in vitro in mesangial cells. Biochem Biophys Res Commun. 2010;391 (4):1585-1591. doi:10.1016/j.bbrc.2009.12.061

40. Shang G, Gao P, Zhao Z, et al. 3,5-Diiodo-l-thyronine ameliorates diabetic nephropathy in streptozotocin-induced diabetic rats. Biochim Biophys Acta. 2013;1832(5):674-684. doi:10.1016/j. bbadis.2013.01.023

41. Zoccali C, Tripepi G, Cutrupi S, et al. Low triiodothyronine: a new facet of inflammation in end-stage renal disease. J Am Soc Nephrol. 2005;16(9):2789-2795. doi:10.1681/ASN.2005040356

42. Abozenah H, Shoeb S, Sabry A, et al. Relation between thyroid hormone concentration and serum levels of interleukin-6 and interleukin-10 in patients with nonthyroidal illness including chronic kidney disease. Iran J Kidney Dis. 2008;2(1):16-23.

43. Xiang GD, He YS, Zhao LS, et al. Impairment of endotheliumdependent arterial dilation in Hashimoto's thyroiditis patients with euthyroidism. Clin Endocrinol. 2006;64(6):698-702. doi:10.1111/ j.1365-2265.2006.02531.x

Diabetes, Metabolic Syndrome and Obesity: Targets and Therapy

Dovepress

\section{Publish your work in this journal}

Diabetes, Metabolic Syndrome and Obesity: Targets and Therapy is an international, peer-reviewed open-access journal committed to the rapid publication of the latest laboratory and clinical findings in the fields of diabetes, metabolic syndrome and obesity research. Original research, review, case reports, hypothesis formation, expert opinion and commentaries are all considered for publication. The manuscript management system is completely online and includes a very quick and fair peer-review system, which is all easy to use. Visit http://www.dovepress.com/testimonials.php to read real quotes from published authors. 